ISAHP 1996, Vancouver, Canada, July 12-15, 1996

\title{
RELATION BETWEEN THE CHOICE PROBABILITY MODEL AND THE AHP MODEL
}

\author{
Toshimasa OZAKI and Nobuji MIYASAKA \\ Supercomputing Center \\ Kansai Research Institute, Kyoto, Japan, 600 \\ ozaki@kyoto.kansai-ri.jp/miyasaka@kyoto.kansai-ri.jp
}

Eizo KINOSHITA

Faculty of Urban Science

Meijo University, Kani, Japan, 509.02

Kei HARA

Faculty of Engineering

Doshisha University, Kyoto, Japan, 610.03

\begin{abstract}
In the Osaka bay area, where many regional development projects have been planed and carried out, and estimates of demand can be dealt with as a problem of qualitative response. The inference of demands requires huge expenses to acquire the necessary microdata, and it is difficult to acquire the factor information in advance.

The AHP (Analytic Hierarchy Process) model is a decision-making tool for use under indefinite conditions. However, inferring demands can not be dealt with it. Behavior of a decision-maker to decide whether to select or not is qualitative in nature. This report defines the choice probability model both for the individual and for a group using information entropy, and attempts a.combination with the choice probability model. The results show that the AHP can be a tool for inferring simply factor information in advance for the choice probability model.
\end{abstract}

\section{Introduction}

In the Osaka bay, where many regional development.projects have been conceptualized, planned and carried out in order to provide functions suitable for Global Metropolis, such as a comfortable residential environment and other excellent facilities. And the infrastructures such as water service, communication line and energy supply institution need to be consolidated. $* \because$.

However, the utility demands and the institution demands in the development projects are too opaque to set the institution capacity using a statistical technique and a computing technique, the inference of demands in these projects has to almost depend on experience and intuition of the participants. Consideräble microdata need to be collected to infer demands, but there are little amounts about the qualitative and quantitative data. In consequence the model frequently remains to the model within the limit of minimum data that be collected. This is the reason why both an analytic tool for inference of demands and an approach way have not been established in ${ }_{n}$ the regional development projects. Therefore it is necessary to infer proper utilization populations in development projects in order to evade overestimation or underestimation of the institutions.

Acknowledgments: The authors wish to thank Mr.Shigeo YAMAMOTO, Mr.Toshimi MUNEHIRA and Mr.Haruo KINOSHITA for many invaluable discussions. 
By the way we need to take into consideration that the economic subjective person such as a consumer and a enterprise are rational in the sense that they give priority to their perceived utility subject to constraints. The AHP (Analytic Hierarchy Process; AHP), also a simple tool for multipurpose decision making, has been developed, gives the relative comparison of demands by positing with an expression of the utility ratio.

However the AHP has not mentioned to the choice probability. In this report, the relation between the choice probability model that is representative in qualitative response models and the AHP model is discussed through intermediate information entropy. And relating the AHP with the choice probability model is confirmed on the basis of the application.

\section{Characteristics of regional development projects and a frame of a valuation research}

1.1 Characteristics of regional development projects in the Osaka bay area

The Osaka bay area is the coastal area including the following zones: Kobe, Osaka, Awaji Island and a part of Tokushima prefecture. It has $290 \mathrm{k} \mathrm{m}$ area whose $30 \%$ is reclaimed ground and had about 144 ten-thousand population in 1985. This area has played an important role in the industry-economy as a prominent heavy and chemical industrial zone after 1900 in Japan, while after an oil shock the industrial structural revolution causes a reduction of population, the efflux of industrial installations and the increasing of the idle grounds.

However, with the construction of Kansai international airport, many development projects are concentrated around Osaka bay to call for expanding and updating harbor and industrial facilities, building new facilities to enhance daily living such as - pleasant parks and areas for international exchanges - and rejuvenating multipurpose areas to enhance opportunities for creativity and social interaction. ${ }^{t} t$

-A total of 103 projects, whose total cost was estimated to amount to120 billion U.S.\$, is being conceptualiżed, planned and carried out. Among thẹm thère are 25 projects which possesses an enterprise scale more than one billion. U.S:\$. And the amúsement type is characteristics of the projects. (in 1994 ; Center for industrial renovation of Kansai).

The model that these projects càn predict súccess-failure is therefore necessary. This research is investigated in regard to the amusement type of projects.

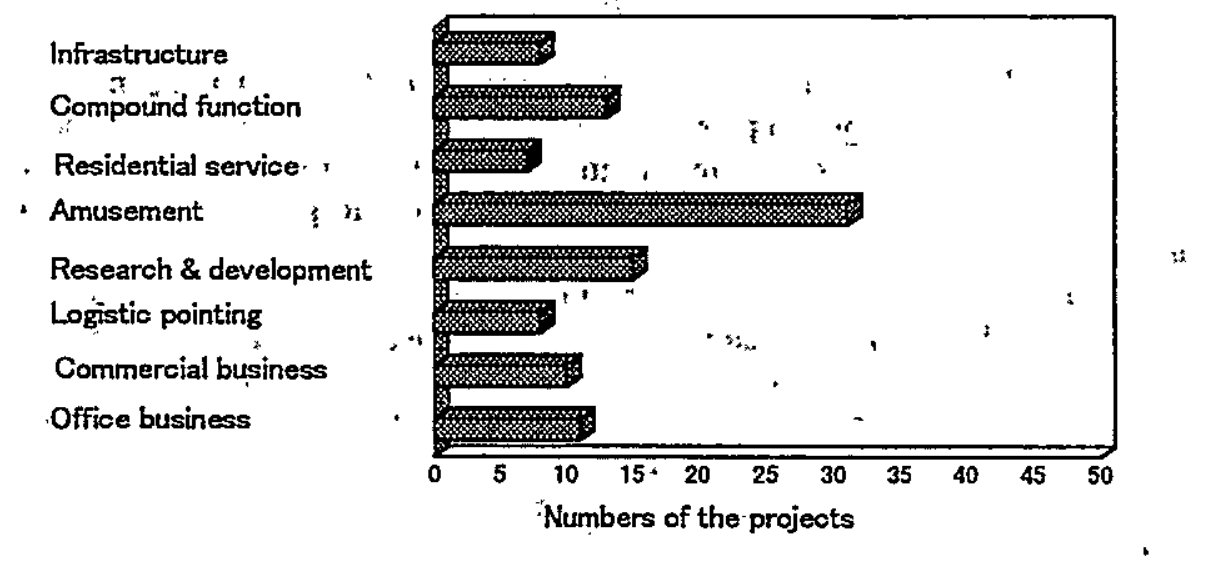

Fig.1 Characteristics of regional development projects in the Osaka bay area

1.2 A frame of the valuation Inferring demands in projects is related to choice behavior of the individual. The 
AHP model can be applied as the technique with which is valued by a decision maker such as a consumer and a enterprise.

Suppose that a decision maker gains any information, from $m$ pieces of the development projects, which is related with the condition of an location (traffic advantage), an admission fee, and so on, with regard to the project, and the priorities are accounted on the basis of information.

In the AHP model, criteria are compared in pairs with respect to their relative weight on a property they share in common. When the value $\mathrm{a}_{\mathrm{ji}}$ is assigned in comparing the criterion $i$ with the criterion $j$, the reciprocal value is assigned to $a_{j i}=1 / a_{j i}$. We shall

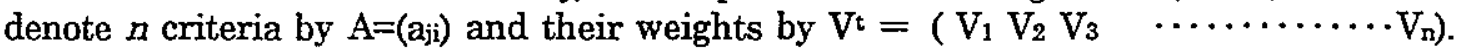
The weight vector $V^{t}$ can be calculated from the maximum eigenvalue of the matrix $A$.

It appears that the decision maker should not order the relative weight prior to making the pairwise comparisons, as the relations between criteria may become complicated.

$$
\left(\begin{array}{ccccc}
a_{11} & \cdots & a_{1 j} & \cdots & a_{2 n} \\
\vdots & \cdots & \vdots & \cdots & \vdots \\
a_{i 1} & \cdots & a_{i j} & \cdots & a_{i n} \\
\vdots & \cdots & \vdots & \cdots & \vdots \\
a_{n 1} & \cdots & a_{n j} & \cdots & a_{m n}
\end{array}\right) *\left(\begin{array}{c}
v_{1} \\
\vdots \\
v_{j} \\
\vdots \\
v_{n}
\end{array}\right)=\lambda\left(\begin{array}{c}
v_{1} \\
\vdots \\
v_{j} \\
\vdots \\
v_{n}
\end{array}\right) \quad(A-\lambda) V=0
$$

$\mathrm{CI}=\left(\lambda_{\max }-n\right) /(n-1)$ is used as a measure of the consistency or reliability of judgments.

However, this method is only applied to evaluate the each priority of the projects. Therefore it is necessary that the AHP model is developed with a choice probability model to estimate the utilization population in the development projects. By the way the priority by the AHP model is often made with grouped persons and the observed data we can obtain is the grouped one with respect to the choice probability. Then let us attempt a combination with the choice probability mode and assume the following frame.

(1) What is the relationship between the AHP model and the choice probability model when an individual person selects only one project from $m$ pieces of the development projects?

(2) What is the relationship between the AHP model and the choice probability model when a grouped persons select only one project from $m$ pieces of the development projects?

The choice probability model is regression model in which dependent variables take place discrete values. So let us discuss the choice probability model by information entropy.

\section{Theoretical equation concerning the combination}

\section{2-1 Choice probability denoted for the individual by information entropy}

Now we will deal with choice behavior of a decision maker in order to infer the demands in regional development projects. Suppose to select only one project out of $m$ pieces of the regional development projects. Let us introduce the choice probability $\mathrm{P}_{\mathrm{i}}$ in the case where the person chooses $i$-project. The utility value of $i$-project for the person is denoted by $\mathrm{V}_{\mathrm{i}}$.

$$
\mathrm{P}_{1}+\mathrm{P}_{2}+\cdots \cdots+\mathrm{P}_{\mathrm{i}}+\cdots=1
$$

Though we do not know which project the person selects, the informational amount received from $i$ project is defined as $-\log P_{i}$ from information theory when $i$-project is chosen practically. Here a unit of the informational amount is essentially same in base of logarithm of either 2 or e. In other words, the informational amount in advance concerning 
the selection of the mean uncertainty which project is to select. Therefore, the choice probability can be thought as a problem of giving and receiving the amount of information which is described using information entropy. When the decision maker chooses $i$-project, the amount of information for decision making is shown as follows, using the informational entropy theory of Shannon.

$$
H=-\sum_{i=1}^{m} P_{i} \log P_{i}
$$

If the choice probabilities are ordered in advance, the decision maker will choose such a project as the expected utility value becomes maximum. However, there is no information concerning either the utility value or the choice probability. For example, if the decision maker repeats to choose $i$-project for one time, $j$-project for two time and so on, he or she will choose such a project as the utility value becomes maximum. That is, it can be thought to perform the selection which makes the expected utility maximum through many kinds of combination of the alternatives. And it is observed from the outside, a series of choosing process is grasped with combination of the system which changes the mean of the expected utility value to maximization. Let us denote the mean of the expected utility value for the person as $E$.

The variation $\mathrm{E}$ of the mean of the expected utility value amounts to the maximum value that the choice probability $P_{i}$ takes numerical value of arbitrary positive and is shown as follows.

$$
\delta \mathrm{E}=\sum_{i=1}^{\mathrm{m}} \mathrm{V}_{\mathrm{i}} \delta \mathrm{P}_{\mathrm{i}}=0
$$

When self-entropy is maximum, that is, all combination are thought about, the variation of Eq. (3) is shown as follows, because it may be thought that decision making is performed.

$$
\delta \mathrm{H}=-\delta\left(\sum_{\mathrm{i}=1}^{m} \mathrm{P}_{\mathrm{i}} \log \mathrm{P}_{\mathrm{i}}\right)=-\sum_{\mathrm{i}=1}^{\mathrm{m}}\left(\log \mathrm{P}_{\mathrm{i}}+1\right) \delta \mathrm{P}_{\mathrm{i}}=0
$$

From Eq. (2) the following equation is obtained.

$$
\sum_{i=1}^{m} \delta \mathrm{P}_{i}=0
$$

The choice probability $P_{i}$ is not independent and follows to from Eq. (2) to Eq.(6) , Eq. (4) and Eq. (6) are multiplied respectively by undetermined coefficient $\alpha$ and $\beta$, and added to Eq. (5), the following equation is obtained.

$$
\sum_{i=1}^{\infty}\left(-\log P_{i}+\alpha+\beta V_{i}\right) \quad \delta P_{i}=0
$$

Since the variations in $\delta \mathrm{P}_{2}$ can occur independently (Appendix 1) and need not to be zero, consequently, to satisfy the above equation each term in summation must be zero as follows.

$$
\log _{i}=\alpha+\beta V_{i}
$$

It is realistic to think that in this case the choice probability also is bigger as the utility value gained from projects is bigger, and the undetermined coefficient $\beta$ has to be positive. Even though Eq. (4) is divided by $\beta$, the result of Eq. (8) does not change. Therefore the coefficient $\beta$ can be set as $\beta=1$. It follows that

$$
\mathrm{P}_{\mathrm{i}}=\frac{\exp V_{i}}{\sum_{i=1}^{m} \exp V_{i}} .
$$

Eq. (9) yields the same form as the called Logit model, it will be defined as the individual Logit model. Hence, this model shall be defined as the choice probability model in convenience in this paper to be dealt with choice behavior of the individual.

$2 \cdot 2$ Basic equation of the combination for the individual 
In the choice probability model, the simplest utility function is represented by the characteristic value $Z_{i j}$ concerning the project $i$ with the content $j$, the form is as follows.

$$
\mathrm{U}_{\mathrm{i}}=\sum_{\mathrm{j}=\mathrm{I}} \beta_{\mathrm{j}} \mathrm{Z}_{\mathrm{ij}}
$$

On the other hand, the evaluated values by the AHP also seems the subjective values concerning a degree of satisfaction. P. Nijkamp has showed the simplest function by using the weight denoted $\lambda$ and project properties $\omega \mathrm{ij}$.

$$
W_{i}=\lambda_{1} \omega_{i 1}+\lambda_{2} \omega_{i 2}+\cdots=\sum_{j=1} \lambda_{j} \omega_{i j}
$$

Then the measured values which are normalized the AHP values are shown as follows.

$$
v_{i}=\frac{H_{i}}{\sum_{i=1}^{m} H_{i}}
$$

But, according to the theory by Luce, the probability of choosing $i$-project from the projects is shown as follows.

$$
x_{1}=\frac{U_{i}^{*}}{\sum_{i=1}^{n} U_{i}^{*}}
$$

Therefore, when $\mathrm{W}_{\mathrm{i}}=U_{\mathrm{i}}^{*}$ is related, the normalized estimations by the AHP amounts to the choice probability of choosing $i$-project. In fact in the AHP, the individual and several participants make the pairwise comparison following prepared categories, for example ,"equal importance ", "moderate importance", "essential or strong importance" and so on. Because a judgment is not uniform, it is natural that this judgment should amount to a probability variable. It has not been defined in terms of $U_{i}^{*}$ that Luce showed. If the logarithmic transformation $\mathrm{U}_{i}=\log U_{i}^{*}$ is applied, the relation between the Luce model and the Logit model for the individual can be shown formally. However, we can not express a standard or cardinal point in the utility value in Eq.(11). Moreover, when the psychophysical rule of Weber-Fechner is only allowed on a relation between amount of sensation and the physical quantities, but, both utilities of the choice probability model and the AHP model are qualitative one which have no validity. Then, we let to show a utility function of the individual Logit model, using the choice probability of the AHP from Eq. (12). Let us investigate the relationship between $W_{i}$ and $v_{i}$, then $W_{i}=\mu{ }^{*} v_{i}\left(v_{i} \neq 0\right.$ $\mathrm{i}=1,2, \cdots, \mathrm{m}$ ) is obtained. Both the utility value of the AHP model and the choice probability Logit model can be expressed by the same parameters, that is $U_{i}=\xi^{*} W_{i}=k v_{1}$. Then the choice probability of the AHP is related to the utility of the choice probability model as follows.

$$
Q=\operatorname{expkv}^{i} / \sum \operatorname{expkv}_{i}-v_{i}=0
$$

2.3 Basic equation of the combination for the group

It seems that the choice probability model of the individual can be applied to that of the group, it is not clear. Now let us clarify the rationality. Now suppose that there are $\mathrm{N}$ decision makers and $m$ choice objects of the regional development projects. Let us introduce the choice probability $\mathrm{P}_{\mathrm{ik}}$ in the case where $k$-th person chooses $i$-project. The utility value of $i$-project for $k$-th person is denoted by $V_{i k}$.

Then according to Appendix 1 and Appendix 2, we can derive the same equation concerning choice probability between the individual and the group. Moreover the grouped expected utility $U_{i}$ is necessary to satisfy $U_{i}=V_{i k}$ for every person, its utility function is the same as that is formed in an individual one. Hence this choice probability model will 
be defined as the grouped Logit model.

\section{Approximate solution}

$3 \cdot 1$ Generation of random numbers

The estimated values by the AHP for $m$ pieces of object projects should fill the following equation.

$$
\mathrm{v}_{1}+\mathrm{v}_{2}+\cdots \cdots+\mathrm{v}_{\mathrm{i}}+\cdots=1 \quad 0 \leq \mathrm{v}_{\mathrm{i}} \leq \mathrm{I}
$$

Random utility ratios to satisfy the above equation are calculated, using the method of linear congruence, of which parameters are adopted by values of Ohkoma. And the method of linear congruence can be regarded as random numbers in practical use.

$$
\mathrm{R}_{\mathrm{n}}=\left(\mathrm{a} \times \mathrm{R}_{\mathrm{n} \cdot \mathrm{1}}+\mathrm{C}\right) \text { MODE } \mathrm{m}
$$

In addition, when number of object projects $m$ become increasing, the periodicity of random numbers occurs. So the other linear congruence with the independent parameter that differs is used in order to evade it. Then the relation between the objective function and the variable is as follows.

$$
\left.Q=\operatorname{Min} \sum e^{\operatorname{expk} v_{i}} / \sum \exp \kappa_{i}-v_{i}\right)^{2}
$$

\section{$3 \cdot 2$ Approximate solution}

In the above equation the coefficient $k$ is calculated as the approximate solution. The project numbers $m, v_{\max }, v_{\min }$ (which is maximum or minimum value in $v_{i}$ ) and a standard deviation $\sigma$, are obtained from the analysis of the AHP, and these variables are approximated with a logarithmic regression equation, assuming the exponential function within the range of project numbers 3 to 20 .

The result of regression analysis is shown in Table 1, the following relationship is

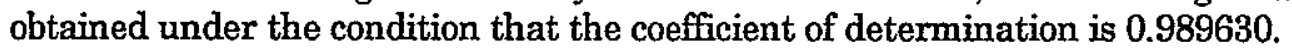

$$
k=2.72536 \mathrm{~m}^{0.500832} \sigma 0.6720268 \mathrm{v}_{\max }-1.191172 \quad \mathrm{v}_{\min } 0.0047912
$$

However the accuracy concerning $v_{\min }$ is still worse, we can not turn down it for the reason why $t$-value is better than $t=2$ may be taken in the case of a social phenomenon and the non-liner equation is approximated with a liner regression.

Table 1 . Result of regression analysis

\begin{tabular}{cccr}
\hline & Coefficient & Deviation & \multicolumn{1}{c}{ t-Value } \\
\hline III & 0.500832 & 0.016793 & 29.823930 \\
$\sigma$ & 0.672068 & 0.031914. & 21.057295 \\
Vmax & -1.191172 & 0.027435 & -43.418280 \\
Vmin & 0.004792 & 0.002799 & 1.711546 \\
Intercept & 1.002602 & 0.026403 & 37.973527 \\
Coefficient of & 0.989630 & & \\
Determination & 180 & & \\
Sample Number & & & \\
\hline
\end{tabular}

\section{Result of the combination of the choice probability model with the AHP model}

$4 \cdot 1$ Valuation of demands analysis by the AHP model

$4 \cdot 1 \cdot 1$ Hierarchy for choosing project 
Inference of utilization population is basic quantity in institution capacities of development projects. Let us decompose the valuation into a hierarchy which provides an overall view of the complex relationships in the situation, and which permits the decision maker to assess whether he or she is comparing issues of the same order of magnitude in

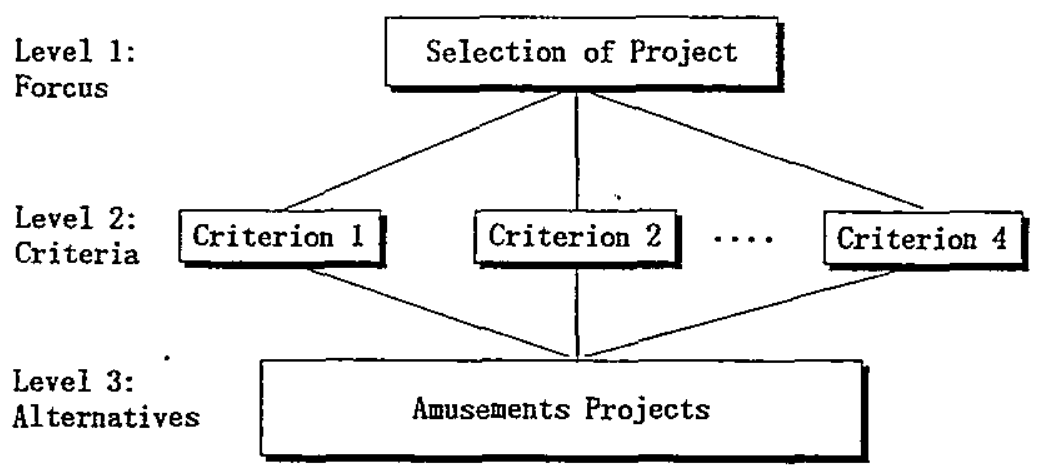

Fig.2. Hierarchy for choosing project

weight or impact on the solution.

On making the hierarchy, the elements should be clustered into four homogeneous groups so they can be meaningfully compared to elements in the next higher level. The only restriction on the hierarchic arrangement of elements is that any element in one level, which must be capable of being related to some elements in the next higher level, which serves as a criterion for assessing the relative impact of elements in the level below.

Finally, we can define the focus as level 1, criteria as level 2 and projects as level 3.

\subsubsection{AHP analysis}

The AHP should be applied to the valuation at the visitors to the amusement institutions. Four parameters have been gained from the individual Logit model by trial and error, that is, existence of an aquarium, existence of a restaurant, an admission fee and a traffic distance. The

\begin{tabular}{|l|c|c|c|c|c|}
\hline & $T$ & $F$ & $R$ & $A$ & Eigen vector \\
\hline Traffic Time (T) & 1 & 4 & 4 & $1 / 4$ & 0.23383 \\
\hline Admission Fee (F) & $1 / 4$ & 1 & 3 & $1 / 6$ & 0.09774 \\
\hline Restaurant (R) & $1 / 4$ & $1 / 3$ & 1 & $1 / 9$ & 0.05018 \\
\hline Aquarium (A) & 4 & 6 & 9 & 1 & 0.61846 \\
\hline
\end{tabular}
ratio is calculated to the existing institution based on these parameters.

The search of time to a station is estimated from the shortest course by train by the method of Warshall-Floyd for each person. Only an admission fee is denoted by yen, and the other parameter is denoted by alternative 1 or 0 , whether it is existed or not. In addition, in order to evade influence by a difference of size of the absolute value, we should define $S_{i j}$ of which $e_{i j}$ is divided by the largest evaluation value eimax with respect to $i$.

$$
\mathrm{S}_{\mathrm{ij}}=\frac{\mathrm{e}_{\mathrm{ij}}}{\mathrm{e}_{\mathrm{imax}}}
$$


It is useful for valuing a complicated problem to utilize a questionnaire and a brainstorming in order to collect lots of opinions, and the latter is adopted in this research.

Table 3. Calculative results

\begin{tabular}{|c|c|c|c|c|c|c|c|}
\hline & Project & $\begin{array}{c}\text { Observed } \\
\text { Value }\end{array}$ & $\begin{array}{c}\text { Estimated } \\
\text { Value }\end{array}$ & $T$ & $F$ & $\mathbf{R}$ & A \\
\hline 1 & A Land & 0.1339 & 0.0640 & 0.784 & 0.333 & 1 & 0 \\
\hline 2 & B Park & 0.0681 & 0.0620 & 0.776 & 0.267 & 1 & 0 \\
\hline 3 & C Land & 0.0660 & 0.0617 & 1 & 0.233 & 0 & 0 \\
\hline 4 & D Park & 0.0489 & 0.0627 & 0.733 & 0.400 & 1 & 0 \\
\hline 5 & $E$ Land & 0.0684 & 0.0611 & 0.733 & 0.333 & 1 & 0 \\
\hline 6 & F Park & 0.0144 & 0.0328 & 0.299 & 0.167 & 1 & 0 \\
\hline 7 & G Park & 0.0181 & 0.0400 & 0.496 & 0.000 & 1 & 0 \\
\hline 8 & H Park & 0.0311 & 0.0336 & 0.383 & 0.000 & 1 & 0 \\
\hline 9 & 1 Land & 0.0299 & 0.0486 & 0.525 & 0.300 & 1 & 0 \\
\hline 10 & J Park & 0.0506 & 0.0504 & 0.264 & 1.000 & 1 & 0 \\
\hline 11 & $\mathrm{~K}$ Land & 0.0527 & 0.0570 & 0.437 & 0.867 & 1 & 0 \\
\hline 12 & L Park & 0.0390 & 0.0554 & 0.548 & 0.533 & 1 & 0 \\
\hline 13 & M Park & 0.0506 & 0.0733 & 0.935 & 0.367 & 1 & 0 \\
\hline 14 & N Park & 0.0303 & 0.0500 & 0.604 & 0.167 & 1 & 0 \\
\hline 15 & 0 Park & 0.0053 & 0.0199 & 0.284 & 0.167 & 0 & 0 \\
\hline 16 & P Aquar ium & 0.2929 & 0.2275 & 0.915 & 0.650 & 1 & 1 \\
\hline
\end{tabular}

\subsection{Combination result}

Now let us verify the combination result of the Logit model with the AHP. Relationship between the utility ratio $v_{i}$ by the AHP and real data Xi about all projects is shown in Fig.3. Based on the evaluated values by the AHP, the coefficients for 16 amusement institutions, $m=16, V \max =0.2275, V \min =0.0199$ and $\sigma=0.0447$, are substituted for Eq.(18), $k=7.74785$ is obtained. Using $U_{i}=k v_{1}$ and Eq.(13), the probability of the grouped Logit model is calculated. The calculated values are in excellent The calculated values are in excellent agreement with the real data on a straight line of $45^{\circ}$. On the diagonal the parameter $k$ yields that the probability of the Logit model is equal to that of the AFIP model.

By the way the revised value is illustrated in order to compare with the original value of the AHP. The revised value is indicated by the interdependence weights using the way of the inner dependence. However, the interdependence intensities which affect the number of visitors are given in a little over $20 \%$, admission fee $6 \%$, restaurant $7 \%$, and aquarium a little over $60 \%$, these values are almost same as the original values of the AHP.

The AHP can be utilized to make an evaluation, whenever it is difficult that a decision maker sets priorities, or whenever no data is sufficient. In that case, though there are many pa

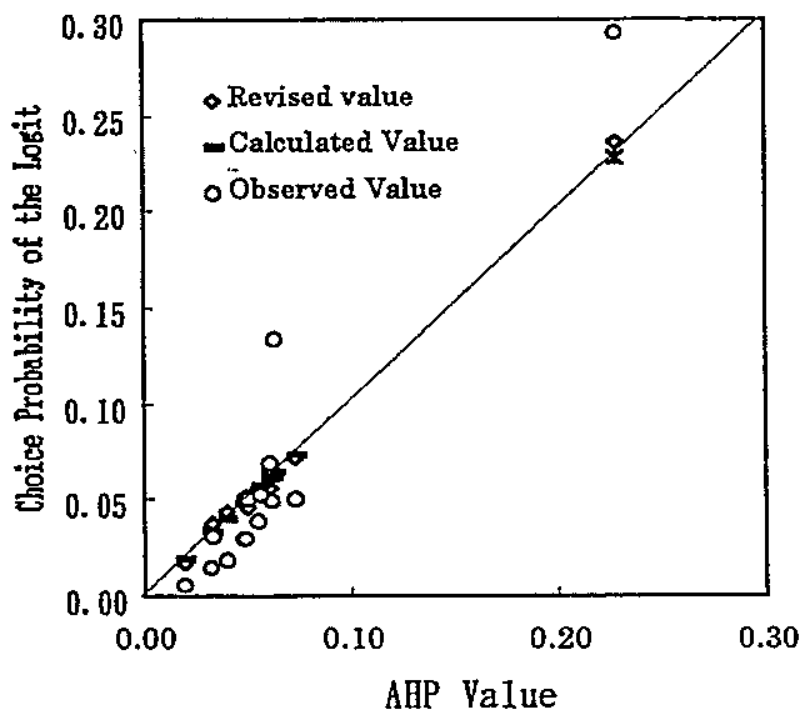

Fig.3 Relationship between AHP and the choice probability model 
rameters to be selected, minor parameters is able to be selected by judgments of plural people. This implys that utility values by the AHP can be utilized in a parameter search in the choice probability of the group.

\section{Conclusion}

It is necessary to infer utilization populations in development projects under indefinite conditions in order to avoid overestimation or underestimation of the institutions, then the Logit model which is a typical QR model is a powerful tool for inferring. However, the Logit model can provide a relationship between cause and effect, it is required huge expenses to acquire the necessary microdata, and it is difficult to acquire the factor parameters in advance.

This paper describes the relation between the Logit model (including the individual Logit and the grouped Logit) and the AHP model which remains in an expression of utility ratio formerly, and the following properties become clear.

(1) The method of information entropy shows that the grouped Logit model becomes the same form as the individual Logit model. Further, it is also clear that the utility function of the AHP is the same form as that of the grouped Logit.

(2) When the utility function is linear, the coefficient $k$ related to the utility function of the grouped Logit model is as follows.

$$
k=2.72536 \mathrm{~m}^{0.500832} \sigma{ }^{0.6720268} \mathrm{v}_{\max }{ }^{-1.191172} \mathrm{v}_{\min } 0.0047912
$$

(3) When factor information does not become clear, or when multitudinous information factors are anticipated, it is hard to presume the information factor within only the Logit model because of worse convergence of a non- linear function. Therefore the AHP can be a tool to infer simply information factor in advance for the Logit model.

(4) The Logit model is required huge expenses to acquire the necessary microdata, and it is desired to the convenient method in view of expenses twin effect. In that case, it is also found that a way of group decision making by a brainstorming is appropriate.

It is regarded useful in a technological examination to a sensory field a demand forecast and so on clear to be able to utilize the AHP as the way factor information of the Logit model and an expected result are presumed in advance.

\section{Appendix 1}

Let us rewrite Eq. (4), Eq. (5) and Eq. (6) respectively.

$$
\begin{aligned}
\delta \mathrm{E}_{\mathrm{k}} & =\sum_{\mathrm{i}=1}^{m} \mathrm{~V}_{\mathrm{ik}} \delta \mathrm{P}_{\mathrm{ik}}=0 \\
\delta \mathrm{H}_{\mathrm{k}} & =-\sum_{\mathrm{i}=1}^{m}\left(\log \mathrm{P}_{\mathrm{ik}}+1\right) \delta \mathrm{P}_{\mathrm{ik}}=0 \\
\sum_{\mathrm{i}=1}^{m} \delta \mathrm{P}_{\mathrm{ik}} & =0
\end{aligned}
$$

Eq. $(A \cdot 1)$ and Eq. $(A \cdot 3)$ are multiplied respectively by undetermined coefficient $\alpha$ and $\beta$, and added to Eq. $(\mathrm{A} \cdot 2)$, the following equation is obtained automatically.

$$
\sum_{\mathrm{i}=1}^{\mathrm{m}}\left(-\log \mathrm{P}_{\mathrm{ik}}+\alpha+\beta \mathrm{V}_{\mathrm{ik}}\right) \delta \mathrm{P}_{\mathrm{ik}}=0 \quad\left(\text { where } \alpha=1+\alpha^{*}\right)
$$

The $\delta \mathrm{P}_{\mathrm{k}}$ are not independent variables, that is, number of $m-1$ are independent and only one is dependent. Let us define $\delta \mathrm{P}_{\mathrm{ni}}$ as the dependent variable, the following equation is obtained from Eq. $(\mathrm{A} \cdot 3)$.

$$
\delta \mathrm{P}_{\text {mk }}=-\sum_{i=1}^{m-1} \delta \mathrm{P}_{i k}
$$

By substituting Eq. $(\mathrm{A} \cdot 5)$ to Eq. $(\mathrm{A} \cdot 4)$ the following equation is obtained. 


$$
\sum_{\mathrm{i}=1}^{\mathrm{z}}\left(-\log _{\mathrm{ik}}+\alpha+\beta \mathrm{V}_{\mathrm{ik}}\right) \delta \mathrm{P}_{\mathrm{ik}}=\sum_{\mathrm{i}=1}^{\pi-1}\left\{\left(-\log _{\mathrm{ik}}+\alpha+\beta \mathrm{V}_{\mathrm{ik}}\right)-\left(-\log _{\mathrm{rkk}}+\alpha+\beta \mathrm{V}_{\mathrm{rk}}\right)\right\} \delta \mathrm{P}_{\mathrm{ik}}
$$

Since the $\delta \mathrm{P}_{\mathrm{ik}}$ in the right term in Eq. (A.6) are independent, each term in summation at the right-hand must be zero. Consequently, $-\log \mathrm{P}_{\mathrm{ik}}+\alpha+\beta \mathrm{V}_{\mathrm{ik}}=$ constant is obtained. Since $\alpha$ is constant multiplier, $-\log \mathrm{P}_{\mathrm{ik}}+\alpha+\beta \mathrm{V}_{\mathrm{ik}}=0$ can be obtained. Namely, the $\delta$ $\mathrm{P}_{\mathrm{ik}}$ can be considered as independent variables

\section{Appendix 2}

A clear way of the choice probabilities of the grouped persons has not been established in the Logit model. We can use basic idea that the choice probability may be grasped with the amount of information.

The summation of amount of information concerning preference of the projects is represented by certain amount of information from all decision makers. From Eq.(9) the following equation obtained to the case of $i$-project.

$$
-\sum_{\mathrm{k}=1}^{\mathrm{N}}\left(\mathrm{P}_{\mathrm{ik}} \log \mathrm{P}_{\mathrm{ik}}\right)(1 / \mathrm{N})=-\mathrm{X}_{\mathrm{i}} \log \mathrm{X}_{\mathrm{i}}
$$

We can calculate $\mathrm{X}_{i}$ with Eq.(A.7) which is non-linear equation and is needed to convergent calculation by the individual choice probability. By Taylor's series method, $X_{i}=(1 / N) \sum_{k=1}^{N} P_{i k}$ is obtained. Substituting it to Eq. (11), $\sum_{k=1}^{N} \sum_{i=1}^{n} P_{i k}\left(V_{i k}-U_{i}\right)=0$ is also obtained. It is necessary to satisfy $U_{i}=V_{i k}$ for every $P_{i k}$, the utility function is the same as that is formed in an individual one.

This method shall be defined as the entropy one. Moreover this method gives the same result of the method of arithmetical mean approximately.

\section{References}

Amemiya T. (1985), Advanced Econometrics, Oxford, Basil Blackwell

Sano S. (1990), Shituteki sentaku bunseki-Riron to Ouyo, Mitsubishi-keizai kenkyusho Morimune K.(1987), Shakaikagaku no keiryoubunseki, Tokyo Daigaku syuppankai Thomas.L.Saaty,(1990), How to make a decision:The Analytic Hierarchy Process, Eur ropiean Journal of Operational Research Thomas.L.Saaty,(1980), The Analytic Hierarchy Process, Mcgrou Hill Luce,(1959), Individual Choice Behavior, John Wiley and Sons Kinoshita Eizo,(1993),AHP Technology and Appication, Sangyou Gyijyutucenter Nijkamp,P. and Delft,A. (1977), Multi-Criteria analysis and Regional Decision-Making, Martinus Nijhoff Social Siences Division, Leiden(Kanzawa Fujioka,Keiso syuppan) Ishitani\&Ishikawa(1993), Shakai systemKougaku, , Asakura syoten Ohkoma .S.,(1983), Kaitei FORTRAN77, Siencesha 